\title{
A fundamental study on photo-oxidative degradation of linear low density polyethylene films at embrittlement
}

\author{
Yu-Chieh Hsu ${ }^{\text {a,b }}$, Michael P. Weir ${ }^{\text {b,c }}$, Rowan W. Truss ${ }^{\text {a,b,* }}$, Christopher J. Garvey ${ }^{\text {b,c }}$, \\ Timothy M. Nicholson ${ }^{\mathrm{a}, \mathrm{b}}$, Peter J. Halley ${ }^{\mathrm{a}, \mathrm{b}}$ \\ a School of Chemical Engineering, The University of Queensland, St Lucia, QLD 4072, Australia \\ ${ }^{\mathrm{b}}$ Cooperative Research Centre for Polymers, 8 Redwood Drive, Notting Hill, VIC 3168, Australia \\ ${ }^{c}$ Australian Nuclear Science and Technology Organisation, Locked Bag 2001, Kirrawee DC, NSW 2232, Australia
}

\section{A R T I C L E I N F O}

\section{Article history:}

Received 15 December 2011

Received in revised form

22 March 2012

Accepted 23 March 2012

Available online 9 April 2012

\section{Keywords:}

Embrittlement

Degradation

Double yield points

\begin{abstract}
A B S T R A C T
Film embrittlement criteria were determined for photo-oxidative degradation of linear low density polyethylene (LLDPE) films by using a range of characterisation techniques: tensile, high-temperature GPC, MAS-NMR, FTIR-ATR, WAXS and SAXS. The key embrittlement criteria was the loss of $95 \%$ elongation at break and the reduction in interlamellar distance, reduced down to approximately $30-50 \AA$, as a result of recrystallisation of mobile short chain fragments produced from chain scission reaction. Interlamellar thinning correlated well with the changes in double yield points seen in the tensile data, where the absence of the second yield point signified that the tie molecules at the lamellar interface underwent chain scission and could no longer transfer the tensile stress to reach c-axis slip of the lamellar crystals. This was also supported by a reduction in amorphous-lamellar interfacial width with ageing time, extracted from SAXS data using the linear correlation function.
\end{abstract}

Crown Copyright $\odot 2012$ Published by Elsevier Ltd. All rights reserved.

\section{Introduction}

The main reactions occurring during photo-oxo-degradation of polyethylene (PE) are well understood by previous studies [1-5]. These are chain scission reactions, crosslinking, oxidation and recrystallisation. The main degradation mechanism is chain scission reaction in the amorphous phase and at the amorphous-lamellar interface [6,7], where oxygen can easily diffuse into these areas [8]. In contrast, the crystalline phase remains mostly inert [9]. Chain scission reaction is initiated by the free radicals generated from the decomposition of hydroperoxide in the presence of UV and oxygen. In particular, tertiary carbon groups (branch points) are more susceptible to chain scission due to the lower dissociation energy of its $\mathrm{C}-\mathrm{H}$ bond compared to unbranched PE chains [10]. Taut tie molecules at the amorphous-lamellar interface are also believed to undergo chain scission during the early stage of UV exposure as they are under stress [11]. However little evidence is shown to prove this theory,

\footnotetext{
* Corresponding author. School of Chemical Engineering, The University of Queensland, St Lucia, QLD 4072, Australia. Tel.: +617 33653729; fax: +617 33654199.

E-mail address: r.truss@uq.edu.au (R.W. Truss).
}

due to the difficulties in measuring tie molecule concentration. Alternate methods to characterise the existence of tie molecules have been considered in this study, that is determining the interfacial width by SAXS data, by using a linear correlation function calculation [12]. In addition, a study of double yield points found in tensile tests should also give information on the tie molecules and the amorphous-lamellar interface. The first yield point has been assigned to the onset of deformation in the amorphous interlamellar phase and the second yield point to the onset of deformation in the lamellar crystals through c-axis slip [13]. Therefore, the absence of a second yield point would demonstrate the lack of tie-chain molecules that would normally transfer the stress in the interlamellar phase on to the lamellar crystals inducing c-axis slip.

Crosslinking also occurs with chain scission reaction, where it is favoured in low oxygen environments [14,15]. Oxidation and recrystallisation are other common reactions seen in UV degraded PE that occur simultaneously with chain scission and crosslinking. These four reactions are considered to contribute over time to the change in overall film properties until eventually the degraded film reaches a point where the film transitions from ductile to brittle.

Film embrittlement is poorly defined in the literature. Scott and Gilead [16] defined the embrittlement point as the exposure time for the PE to lose $95 \%$ of its original elongation to break while in other studies [11,17], an arbitrary loss of $50 \%$ of the original 
elongation to break has been used. Fayolle et al. [11] recognised that the embrittlement point was when the polymer property transitioned from ductile to brittle. Fayolle et al. [1] suggested that embrittlement occurs when the interlamellar thickness was 60-70. . They argued that at this point, the lamellar crystals are unable to deform either because of physical constraint or because of scission of tie molecule between the lamellae. Other criteria, like lamellar thickness or long period, were not used because they were dependent on thermal history. However, Fayolle et al.'s [1] embrittlement criteria were based on thermally degraded HDPE. Other studies have tried to link film embrittlement with oxidation, where one study [17] claimed the carbonyl index (CI) at embrittlement is 0.3 , but others $[18,19]$ found no relationship. The $\mathrm{CI}-\mathrm{embrittlement} \mathrm{relationship} \mathrm{is} \mathrm{clarified} \mathrm{in} \mathrm{this} \mathrm{study.}$

No work has previously examined the photo-oxo-degradation of commercial LLDPE. This study aimed at defining a film embrittlement criteria for photo-oxidative degradation of LLDPE by correlating the mechanical, molecular chain, crystal morphology and chemical properties at the point when PE transitions from being ductile to brittle. A double yield points study and SAXS/WAXS interfacial width was also used to understand the changes in tie molecule at the interlamellar-lamellar interface.

\section{Experimental}

\subsection{Materials}

The base LLDPE studied was Dowlex 2045; manufactured by The Dow Chemical Company and synthesised by a Zielger-Natta catalyst with octene-1 co-monomer [20]. Two sets of cast Dowlex 2045 LLDPE films were studied, where one set was aged in a QUV (an accelerated UV ageing device) immediately after processing (ZNLLDPE), but the other was stored in an ambient and dark environment for a six month period, then aged in a QUV (stored ZN-LLDPE).

\subsection{Film processing}

Films were cast extruded at Queen's University Belfast (QUB, Belfast, United Kingdom) to $50 \pm 3 \mu$ m thickness, using a Dr Collin cast film/sheet extrusion line Ex-25-M, with a $25 \mathrm{~mm}$ screw diameter, through a coat hanger die at $30 \mathrm{rpm}$ screw speed, $205^{\circ} \mathrm{C}$ melt temperature and 88 bar melt pressure.

\subsection{UV ageing}

ZN-LLDPE cast films were aged in a QUV, following a standard ageing method [21]. The QUV used was a Q-Panel first generation model (1992) that did not have irradiance control and was installed with Q-Lab UVA-340 lamps. The film samples were $55 \mathrm{~mm}$ in distance from the UV lamps, and the humidity cycle was not utilised. The QUV irradiance was measured using a CR-10 radiometer and found to have an average irradiance of $0.77 \pm 0.01 \mathrm{~W} / \mathrm{m}^{2} \mathrm{~nm}$ $340 \mathrm{~nm}$. Samples were aged in the QUV for various time intervals, up to 92 days.

\subsection{Mechanical properties by tensile testing}

Tensile tests were carried out on an Instron 5584, operating with Blue-Hill 2000 software. Samples were prepared into $22 \mathrm{~mm} \times 200 \mathrm{~mm}$ rectangular strips by following ASTM D6287 [22], and tensile tests were carried out at $250 \mathrm{~mm} / \mathrm{min}$ between two rubber faced grips with $25 \mathrm{~mm}$ gauge length, at $23{ }^{\circ} \mathrm{C}$ and $50 \%$ humidity (ASTM D288 [23]). Eight to ten samples were performed for each sample type in both the extrusion or machine direction (MD) and in the transverse (to the extrusion) direction (TD).

\subsection{Molecular weight distribution by high-temperature gel permeation chromatography}

Samples were dissolved in 1, 2, 4 tri-chlorobenzene (stabilised with $200 \mathrm{ppm}$ butylated hydroxytoluene/ionol) for approximately $2 \mathrm{~h}$ at $165^{\circ} \mathrm{C}$ under gentle stirring, then filtered over a $0.5 \mu \mathrm{m}$ filter. The solid content trapped in the mesh was dried under vacuum at $150{ }^{\circ} \mathrm{C}$, cooled then weighed. This solid mass was an estimate of gel content. The standard method to determine gel content, ASTM D2765, uses boiling decahydronaphthalene or xylene to dissolve ethylene plastics for 6 and $12 \mathrm{~h}$, respectively [24]. Although the solvent used in this gel-content experiment was not recommended by the standard procedure, and the extraction time was much shorter, the gel content values provided a higher estimation of crosslinking PE molecules. The variation in experimental method was taken into account in the result analysis.

A Waters GPCV2000 GPC system was used. It was set-up with three mixed B Polymer Laboratories PL-Gel (10 $\mu \mathrm{m}$ particles) columns and an IR4 concentration detector (PolymerChar, Valencia, Spain) to measure molecular weight. The injection volume was $218 \mu \mathrm{L}$ and the GPC injector, columns and detector were operating at $145{ }^{\circ} \mathrm{C}$.

\subsection{Oxidised species characterised by solid-state nuclear magnetic resonance - cross-polarisation magic-angle spinning (MAS-NMR)}

Solid-state NMR was conducted on a Bruker Avance III spectrometer with a $300 \mathrm{MHz}$ magnet, $4 \mathrm{~mm}$ zirconia rotor, operated at $20{ }^{\circ} \mathrm{C}$ at a $5 \mathrm{kHz}$ spinning rate. A cross-polarisation with magicangle spinning and high-power decoupling method was used, with a contact time of $1 \mathrm{~ms}$, decoupling method - tppm 15, of relaxation delay $3 \mathrm{~s}$ and 20,000 scans were collected.

\subsection{Oxidised species characterised by Fourier transform infrared- attenuated total reflectance spectrometry (FTIR-ATR)}

A Thermo Nicolet 5700 Diamond ATR was used to obtain FTIR spectra in the range $500-4000 \mathrm{~cm}^{-1}$ at room temperature. It was set at $4 \mathrm{~cm}^{-1}$ of resolution and each spectrum was averaged over 64 scans. Five to six FTIR-ATR spectra were taken for each sample. The carbonyl index $(\mathrm{CI})$ was calculated by the integral of the carbonyl peak $\left(1650-1800 \mathrm{~cm}^{-1}\right)$ and the $\mathrm{C}-\mathrm{H}$ peak $\left(1420-1480 \mathrm{~cm}^{-1}\right)$, measured above a common baseline (Equation (1)) $[4,17,25,26]$.

$\mathrm{CI}=\frac{\text { Absorption of carbonyl species }}{1650-1800 \mathrm{~cm}^{-1}}$

\subsection{Degree of crystallinity and crystal morphology by SAXS and WAXS}

X-ray scattering experiments were carried out at the Australian Synchrotron small- and wide-angle scattering (SAXS/WAXS) beamline (Australian Synchrotron, Clayton, Victoria, Australia). Film samples were mounted on a 2-dimensional translation stage on a plate with a regular grid of holes, stuck down at the edges using tape such that the films were perpendicular to the beam but not under strain. SAXS and WAXS images were collected using a MAR CCD detector, and the scattering intensity was normalised for exposure time, transmission and sample thickness. The sample to detector distance for each configuration was calibrated using a silver behenate standard sample.

The WAXS configuration used a beam energy of $20 \mathrm{keV}$ and a sample-detector distance of $318 \mathrm{~mm}$ giving a $Q$ range of $0.1-3.8 \AA^{-1}$ (where $Q=(4 \pi / \lambda) \sin \theta, 2 \theta$ is the scattering angle, and $\lambda$ 
is the wavelength of the X-ray radiation). The samples were exposed for $10 \mathrm{~s}$ per image.

The SAXS configuration used a beam energy of $10 \mathrm{keV}$ and a sample-detector distance of $1540 \mathrm{~mm}$ giving a $Q$ range of $0.09-0.135 \AA^{-1}$. The samples were exposed for $2 \mathrm{~s}$ per image.

WAXS images were processed using Fit2D analysis software. The background scatter due to the air taken through an empty position on the sample grid was subtracted and an appropriate mask was used to exclude scattering from around the beamstop. Radial integration was performed to give one-dimensional data in the form of intensity $(I)$ vs. $Q$. The 1 -D data were processed using Igor Pro's Multi-peak Fit function to extract the degree of crystallinity using Equation (2).

$\%_{\mathrm{C}, \mathrm{W}}=\frac{A_{\mathrm{C}}}{A_{\mathrm{A}}+A_{\mathrm{C}}}=\frac{A_{(110)}+A_{(200)}}{A_{\mathrm{A}}+A_{(110)}+A_{(200)}}$

where $A_{\mathrm{C}}$ and $A_{\mathrm{A}}$ are the total area of crystalline and amorphous peaks respectively, and $A_{(\mathrm{hkl})}$ is the area of the peak (hkl). The crystallite sizes $L_{(\mathrm{hkl})}$ for the (110) and (200) peaks were estimated using the Scherrer's equation (Equation (3)).

$L_{\mathrm{hkl}}=\frac{k^{\prime} \lambda}{\beta_{0} \cos \theta}$

where $k^{\prime}$ is the shape factor (assumed equal to 1 ), $\lambda$ is the X-ray wavelength, $\beta_{0}$ is the angular broadening in radians and $\theta$ is the scattering angle (half of the $2 \theta$ value). No correction in $\beta_{0}$ was made for the instrumental broadening which was assumed to be negligible in comparison to the size broadening.

SAXS images were processed using the SAXS15ID software by subtracting for air scatter and masking around the beamstop. Radial integrals of the scattering patterns were calculated. The 1-D scattering profiles were processed using the software CORFUNC [27] to calculate and analyse the Linear Correlation Function (LCF, $\gamma_{1}(x)$ ) [12] to extract a number of key structural parameters such as lamellar long period (LP), lamellar thickness $(L)$, and the interfacial width $(E)$ between the crystalline and amorphous phases.

\section{Results and discussion}

\subsection{Mechanical properties}

The mechanical properties of ZN-LLDPE films are key criteria to determine film embrittlement. Figs. 1 and 2 show the average elongation at break and average Young's modulus, respectively, for stored ZN-LLDPE film in both MD and TD. Only one aged (unstored) ZN-LLDPE film (UV 19 days) was tensile tested due to insufficient amount of samples (Fig. 3). However, when comparing the stress-strain curves of stored and unstored ZN-LLDPE films, the yielding behaviour of ZN-LLDPE films showed similar trends with ageing time.

Stored ZN-LLDPE films showed significant decrease in ductility after 7 days of UV exposure and after 21 days, it had lost over $95 \%$ of its original elongation at break. The elongation at break decreased and the modulus gradually increased with UV ageing. The high Young's moduli values of highly UV aged films were characteristic of brittle materials.

The embrittlement point has traditionally been defined in terms of a specific loss of elongation to break. The data in Fig. 1 shows that the elongation to break follows an approximately S-shaped curve with exposure time. The inflection point of the S-shaped curve is at approximately $50 \%$ loss of the initially elongation to break although at this point, the films still have significant ductility. A better indication of embrittlement is given by the lower shelf of the S-

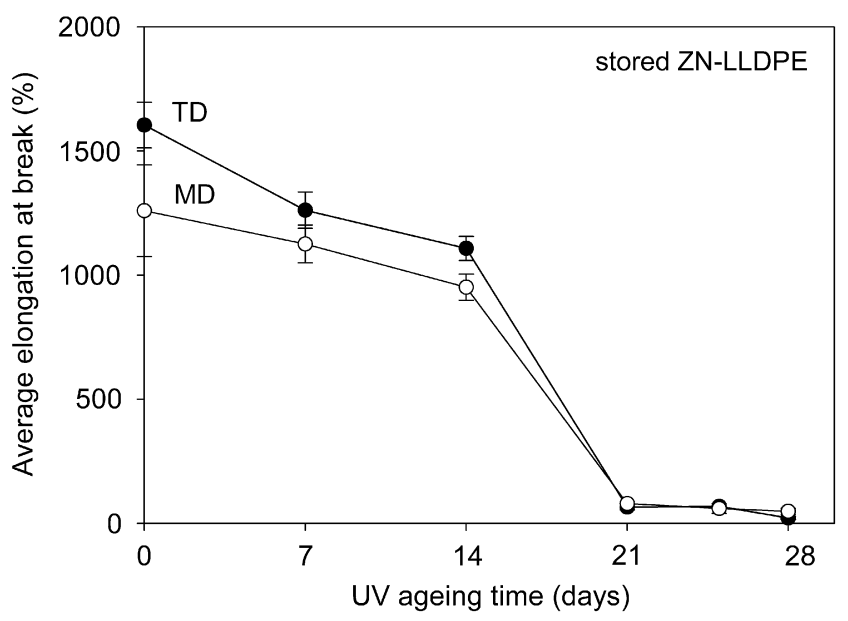

Fig. 1. Average elongation at break for stored ZN-LLDPE cast films with UV ageing time, in machine (MD) and transverse direction (TD), $n=8-10$, error bars = standard deviation.

shape curve. This region is reached after approximately $95 \%$ loss of the initial elongation to break. Film embrittlement can be very pragmatically simulated by handling the film and finding the point when the film breaks with a gentle tug of the film. Although arbitrary, the 'finger poke' test suggested brittleness occurs some 7-10 days longer than the point where the material had lost $95 \%$ of its initial elongation to break in the tensile test. Consequently, Scott and Gilead's [16] definition using the 95\% loss of initial elongation to break will be used in this paper. The relationship of this point to structural parameters in the polymer and deformation mechanisms will be explored in subsequent sections.

ZN-LLDPE films reached embrittlement after 56-70 days. Stored ZN-LLDPE embrittled after 21 days, which was approximately three times faster than unstored films. This suggested that a six month storage period had a significant effect on the degradability performance of ZN-LLDPE. Prior to storage, ZN-LLDPE films were exposed to UV, heat and oxygen for a short period of time (during polymerisation and/or processing) that could have led to the formation of hydroperoxides and free radicals. While the films were stored, these hydroperoxides could decompose and the free radicals

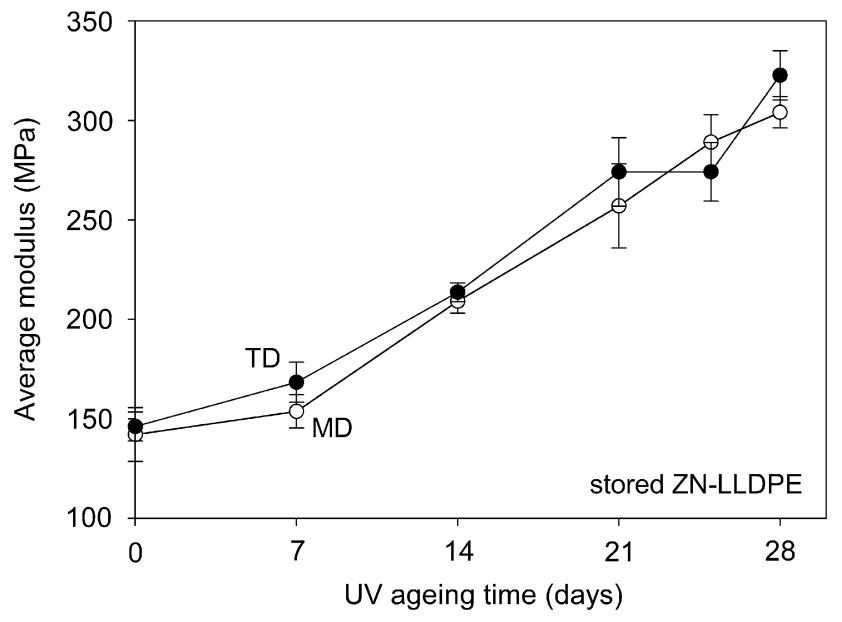

Fig. 2. Average Young's modulus for stored ZN-LLDPE cast films with ageing time, in machine (MD) and transverse direction (TD), $n=8-10$, error bars = standard deviation. 

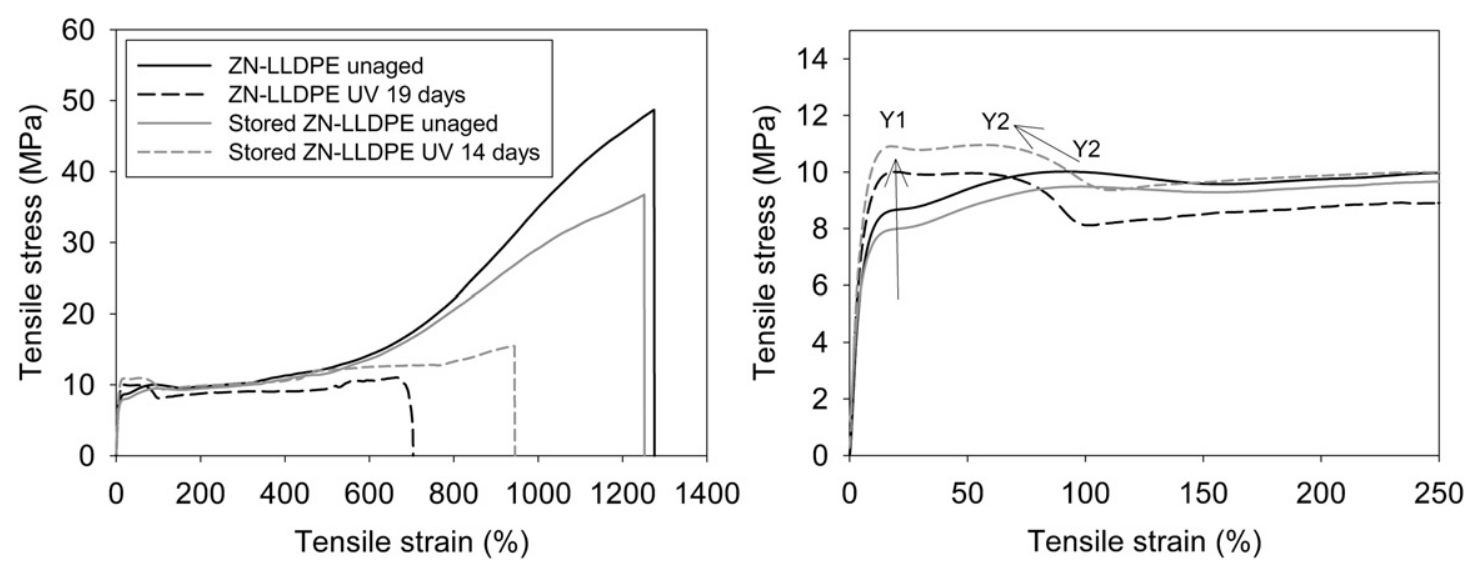

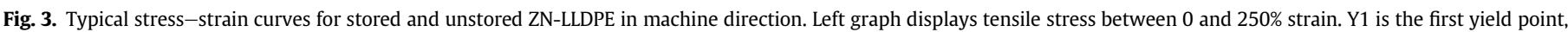
$\mathrm{Y} 2$ is the second yield point and arrows depict the yield point trend.

initiated chain scission reaction. Later, when exposed to UV in the QUV, this accelerated the photo-oxo-degradation of PE.

\subsection{Molecular weight distribution}

The weight-average molecular weight (Mw) and numberaverage molecular weight (Mn) of ZN-LLDPE films decreased dramatically with UV ageing time, and the polydispersity (PDI) and gel content gradually increased with ageing time (Table 1 ).

The average Mw of unaged ZN-LLDPE was 108,700 $\pm 5440 \mathrm{~g} / \mathrm{mol}$ and it decreased to approximately $20,000 \mathrm{~g} / \mathrm{mol}$ at film embrittlement (between 56 and 70 days). The decrease in average Mw was due to chain scission reaction. Although there were some crosslinking reactions, as shown by the gel content increase with ageing, the dominant photo-degradation reaction was chain scission reaction, in the presence of oxygen and UV [14,28].

Fayolle et al. [11] defined one of the criteria for embrittlement as a critical molecular weight range, 40,000-100,000 g/mol, calculated by viscosity-shear rate curves of thermo-degraded PE. Fayolle's values were much higher than those found for photo-oxodegraded ZN-LLDPE, which could be due to discrepancy in the definition of embrittlement at 50\% elongation at break, differences between thermo- and photo-degradation (additional crosslinking) and the characterisation method.

The increase in PDI suggested that chain scission reaction increased the number of lower molecular weight species as well as the heterogeneity in the PE system, due to crosslinking and oxidation (shown by MAS-NMR and FTIR-ATR).

\subsection{Oxidative products}

Solid-state magic-angle spinning nuclear magnetic resonance (MAS-NMR) spectra for UV aged and unaged ZN-LLDPE are shown in Fig. 4. The main oxidative products formed were secondary

\section{Table 1}

Number-average and weight-average molecular weight, polydispersity and gel content of ZN-LLDPE films with increase UV ageing time.

\begin{tabular}{lrrrl}
\hline $\begin{array}{l}\text { UV ageing } \\
\text { time (days) }\end{array}$ & Mn (-) & Mw (g/mol) & PDI (-) & Gel content (\%) \\
\hline 0 & 29,700 & 108,700 & 3.66 & n/a \\
56 & 6890 & 26,800 & 3.90 & 13.30 \\
70 & 4320 & 17,900 & 4.15 & 12.90 \\
90 & 2240 & 9100 & 4.08 & 22.00 \\
\hline
\end{tabular}

alcohols (72 ppm), alkenes (vinyl groups) (114 and $138 \mathrm{ppm})$, and esters and acids (172-184 ppm), and the hydroperoxides (85 ppm) were believed to decompose to form these oxidative product, based on a gamma-radiated PE study by Assink et al. [29]. However, the signal to noise in MAS-NMR spectra was too low to clearly determine this. The low signal to noise ratio in MAS-NMR spectra also made it difficult to make a quantitative study. Alternatively this was done using FTIR-ATR by calculating the carbonyl index.

FTIR-ATR was used in conjunction with MAS-NMR to quantify the degree of oxidation in ZN-LLDPE films over the ageing period. Similar oxidative products were observed in FTIR-ATR as to MASNMR; these were carbonyl, vinyl and ester groups.

The carbonyl index (CI) values for stored and unstored ZN-LLDPE are shown in Fig. 5. The CI for stored and unstored ZN-LLDPE increased at a similar rate, which shows that the photo-oxidative degradation of ZN-LLDPE was determined by its UV exposure time and the additional storage period did not affect its photo-oxodegradability. This result did not match with the tensile results (i.e. the number of days until embrittlement), which suggest that the mechanical and oxidation properties do not correlate. The average $\mathrm{Cl}$ at embrittlement point was approximately 0.5 (56 days) for unstored and 0.1 (21 days) for stored ZN-LLDPE, which was significantly different to the value determined in Naddeo et al. [17] of 0.3 . This further shows that there was a weak correlation between the degree of oxidation and embrittlement. Similar results were found by Geetha et al. [18] and Liu et al. [19].

The carbonyl index was not a reliable indication of degradation for a few reasons. $\mathrm{CI}$ indeed measures oxidised species but the chain scission process does not solely terminate with oxygen groups but with crosslinking. Secondly, the oxidation process begins on the surface and free radicals propagate towards the centre of the sample leading to further degradation. The ATR technique can only analyse $1-2 \mu \mathrm{m}$ deep from the sample surface, which limits the total oxidative products measured and consequently may not represent the bulk sample that was $50 \pm 3 \mu \mathrm{m}$ thick. This would become less significant for thinner film samples. Alternatively, transmission mode was used, but very thin films were required to achieve a substantial signal.

\subsection{Degree of crystallinity and crystal morphology}

The degree of crystallinity for ZN-LLDPE film measured via WAXS is listed in Table 2. The degree of crystallinity increased gradually with ageing time, starting from 34.7\% (unaged) and increased to $51.8 \%$ at film embrittlement (56 days). 


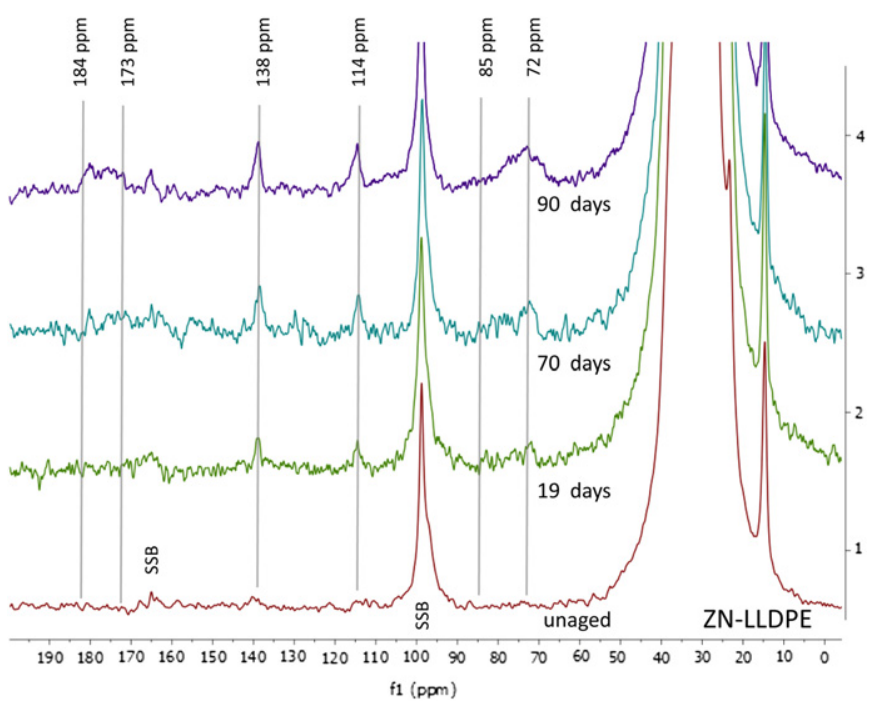

Fig. 4. MAS-NMR spectra for ZN-LLDPE from unaged to 90 days exposed in the QUV (bottom to top); SSB, spinning sidebands; secondary alcohol $=72 \mathrm{ppm}$, hydroperoxides $=85 \mathrm{ppm}$, alkenes $=114,138 \mathrm{ppm}$, esters, acids $=173-184 \mathrm{ppm}[29]$.

Fig. 6 shows crystallite size calculated using the Scherrer's equation for the (110) and (200) WAXS peaks as a function of UV age. This shows that the size of the crystallites in these directions increased significantly from unaged to 14 days in UV, but showed no significant difference thereafter. The crystallite size was a measure of the length scales over which the crystals scatter coherently. This implies that the average length scales over which the samples are perfectly crystalline increases with age. It also implies that the existing crystallites do not break apart with increasing age.

The increase in crystallinity and crystal size was the result of recrystallisation. Over time, the mobile small chain fragments formed from chain scission reaction, underwent reorganisation and re-crystallised. Recrystallisation is a common outcome of chain scission and has been observed in previous studies of photodegraded PE [30-32]. It was interesting to see that in a degraded PE system, PE chain lengths are becoming more heterogeneous

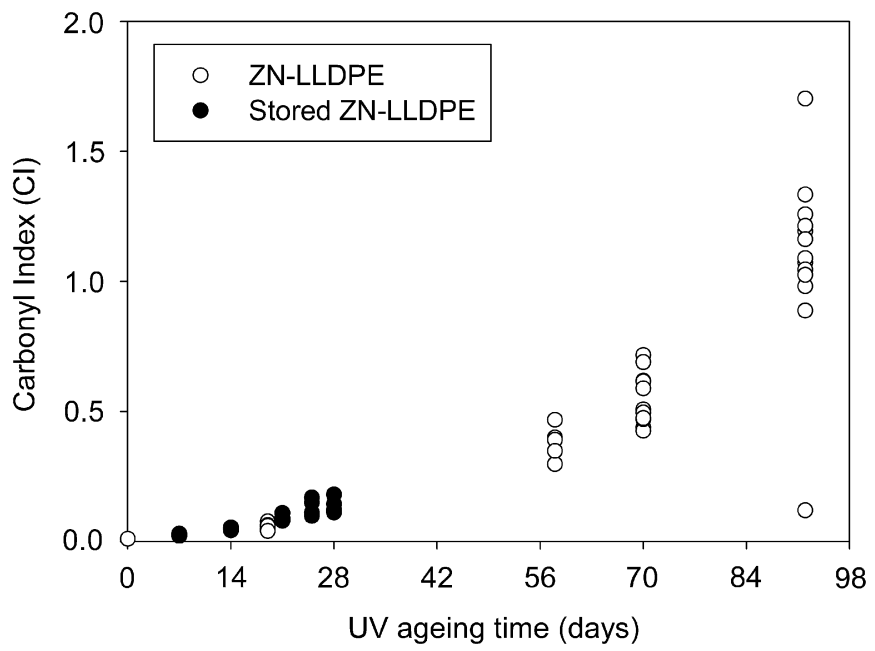

Fig. 5. Carbonyl index $(\mathrm{CI})$ as a function of ageing time for stored and unstored $\mathrm{ZN}$ LLDPE.
Table 2

Degree of crystallinity as extracted from WAXS data for ZN-LLDPE films, as a function of UV age.

\begin{tabular}{ll}
\hline $\begin{array}{l}\text { UV ageing time } \\
\text { (days) }\end{array}$ & $\begin{array}{l}\text { WAXS degree of } \\
\text { crystallinity (\%) }\end{array}$ \\
\hline 0 & 34.7 \\
14 & 46.1 \\
21 & 47.2 \\
42 & 48.9 \\
56 & 51.8 \\
\hline
\end{tabular}

(increasing in PDI, forming oxidative products and crosslinked species) but the molecular chains were finding a balance of order through recrystallisation. The crystallinity increase correlated well with the increase in Young's modulus observed in the tensile data (Fig. 2).

The SAXS spectra for ZN-LLDPE are shown in Fig. 7, where the asterisk marks the position of a peak present in unaged films of unknown origin. It is believed that this peak is attributed to a surfactant or oil contaminant from the cast extrusion process, as other Dowlex 2045 LLDPE films made using a film blowing tower did not show the same peak. This contaminant is considered a volatile compound, and hence is not present once exposed to UV. Fig. 8 shows the LCF $\gamma_{1}(x)$ as a function of UV ageing time for the data shown in Fig. 7. SAXS data is presented as $I^{*} Q^{2}$ which includes the Lorentz correction to take into account the random orientation of the lamellae. The clearest direct observation from $\gamma_{1}(x)$ is that the second maximum, indicating the long period of the periodic semi-crystalline structure gets shorter with increasing UV age. Further parameters are extracted mathematically within the CORFUNC software and this process is described in detail elsewhere [27].

The lamellar long period (LP) and lamellar thickness ( $L)$ as extracted from SAXS data analysed using LCF are shown in Fig. 9 and the interfacial width between the lamellar crystals and the interlamellar phase as a function of UV age is shown in Fig. 10.

Lamellar thickness for ZN-LLDPE showed no significant change with UV ageing, but the long period decreased gradually from $137 \pm 10 \AA$ (unaged) and reached $98 \pm 10 \AA$ at film embrittlement (56 days) (Fig. 9). This in turn means the interlamellar spacing (long period subtract lamellar thickness and two interfacial widths) has

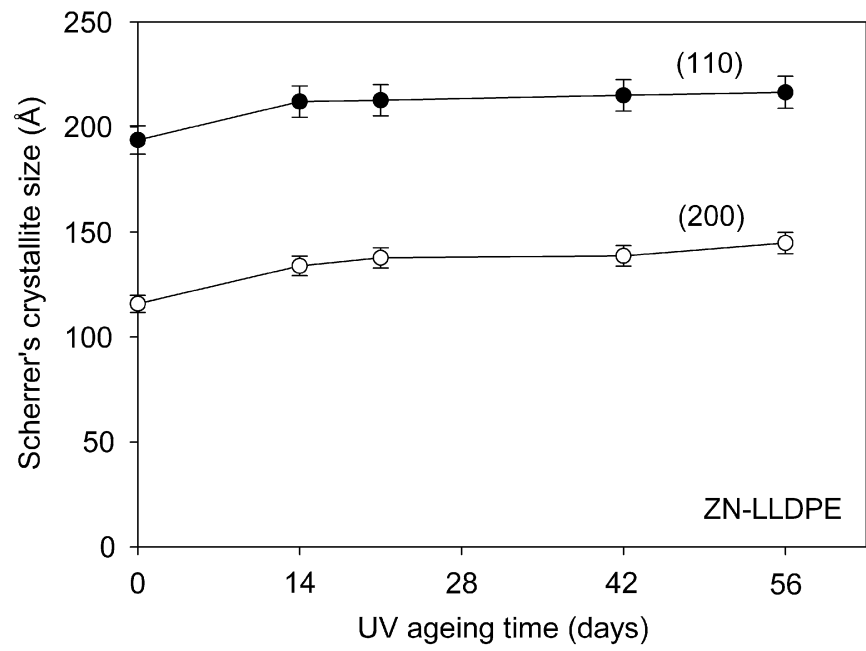

Fig. 6. Crystallite size calculated using the Scherrer's equation for the (110) and (220) WAXS peaks as a function of UV age for ZN-LLDPE films. 


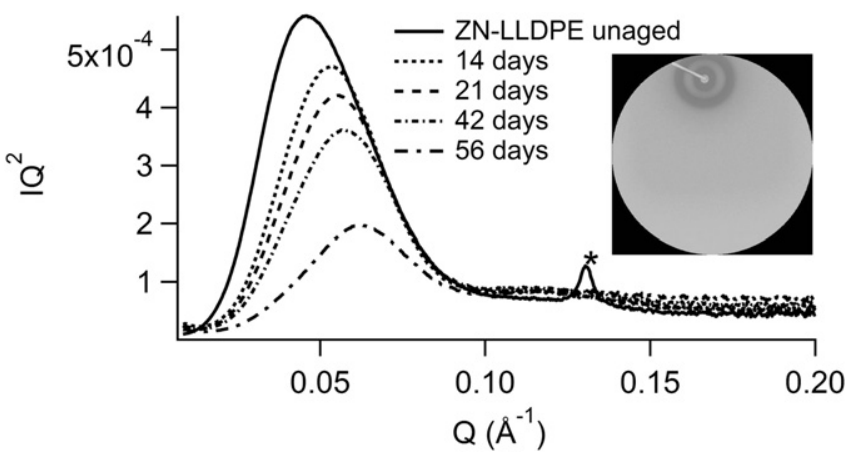

Fig. 7. SAXS data from ZN-LLDPE film with a range of UV ageing times from unaged to 56 days, presented as $I^{*} Q^{2}$ vs. $Q$. The asterisk marks the position of a peak present in unaged films of unknown origin. Inset: a typical SAXS image from the MAR detector.

decreased from $81 \pm 7 \AA$ (unaged) to $42 \pm 9 \AA$ (56 days). The interlamellar thickness at film embrittlement was at a similar scale to Fayolle et al.'s [11] embrittlement criterion of $60-70 \AA$ interlamellar thickness. The interlamellar thinning occurred as a result of chain scission reaction and recrystallisation in the amorphous phase (Fig. 9). The decrease in molecular chain lengths, due to chain scission reaction, increased chain mobility and the chains recrystallised. The PE films shrank with UV ageing, indicating an increase in density. Control PE films thermally aged at the same temperature as that experienced during UV ageing did not show any increase in the degree of crystallinity indicating that the densification was a result of exposure to UV. Moreover, SAXS data showed a densification of the amorphous phase (signified by the decrease in scattering intensity with age in Fig. 7 and the increase in the first minimum of the LCF with age in Fig. 8) and such densification would be expected to result in a decrease in the long period.

The interlamellar amorphous phase is the phase that contributes to the film's ductile property [13]. However, with interlamellar phase recrystallising with ageing time, this results in the ZN-LLDPE film becoming more crystalline and is one of the attributes to film embrittlement. Fig. 10 shows there is a gradual decrease in interfacial width with ageing time, where it is estimated to be $4.4 \pm 0.3 \AA$ (unaged) and decrease to $1.2 \pm 0.1 \AA$ after 42 days in the QUV and increase back to $2.8 \pm 0.3 \AA$ after 56 days (film embrittlement). The interfacial width $(E)$ represents the length scale over which the electron density varies between the crystalline and amorphous regions of the films. This corresponds to an area occupied by partially confined molecules (i.e. loop and tie molecules) that are neither fully crystalline nor fully amorphous. A net decrease in $E$

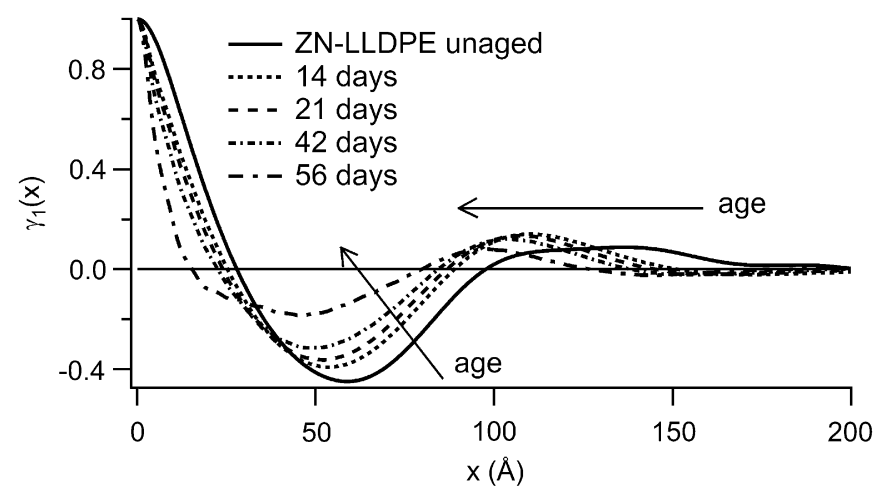

Fig. 8. For ZN-LLDPE films: Linear correlation function as a function of UV ageing time.

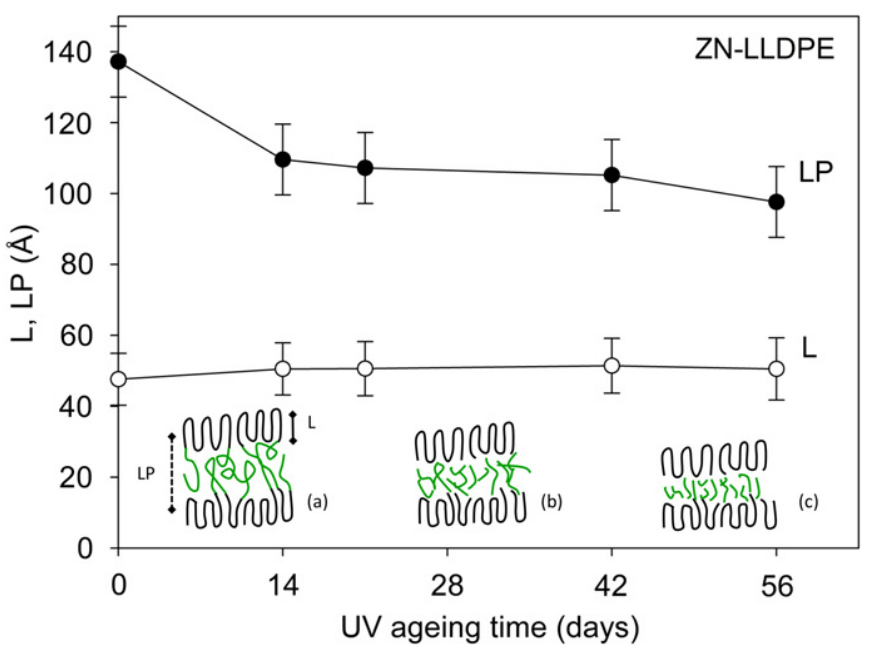

Fig. 9. Lamellar long period (LP) and lamellar thickness $(L)$ as extracted from SAXS data for ZN-LLDPE film. The interlamellar spacing or amorphous thickness $S=\mathrm{LP}-(L+2 E)$; (a) unaged, (b) undergoing chain scission and recrystallisation, (c) significant reduction in interlamellar thickness due to recrystallisation; error bars $=$ standard error.

may be interpreted as evidence for chain scission within (but not confined to) this interfacial region, and that in turn suggests that loop or tie molecules could be cut and their fragments incorporated either into the crystalline or amorphous phases, creating a sharper interface. However, $E$ could be broadened by crystal imperfections in the re-crystallised amorphous phase (caused by bulky oxygen groups and crosslinked species), which may explain the increase in $E$ between 42 and 56 UV ageing days.

\subsection{Double yield points}

The typical tensile stress-strain curves for stored ZN-LLDPE are plotted in Fig. 11. These show the two yield points characteristic of polyethylene, labelled Y1 for the first yield point and Y2 for the second yield point. The tensile stress and tensile strain of these two yield points changed with ageing time, and these values are plotted in Fig. 12.

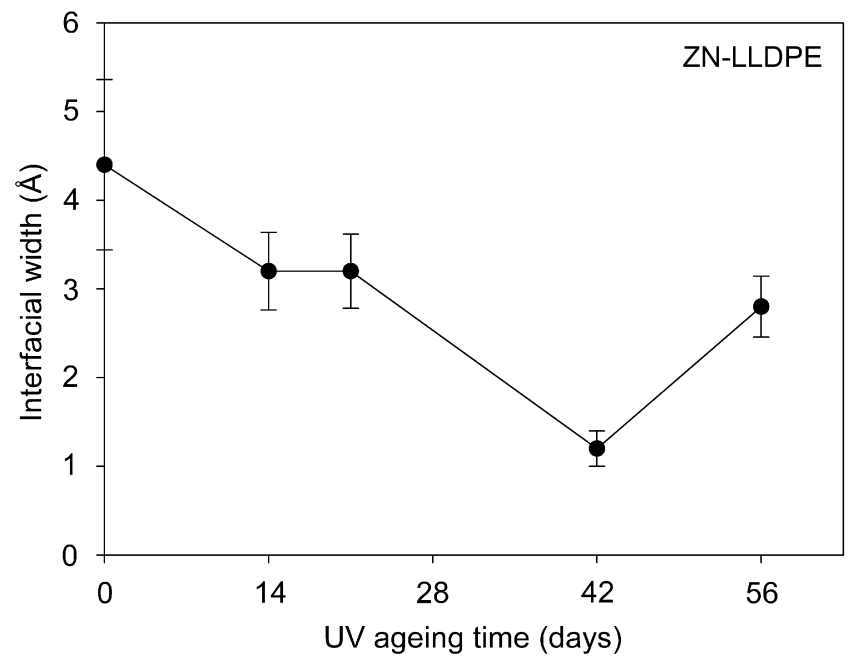

Fig. 10. Interfacial width $(E)$ between crystalline and amorphous regions as a function of UV age for ZN-LLDPE film, as extracted from SAXS data using the LCF; error bars $=$ standard error 

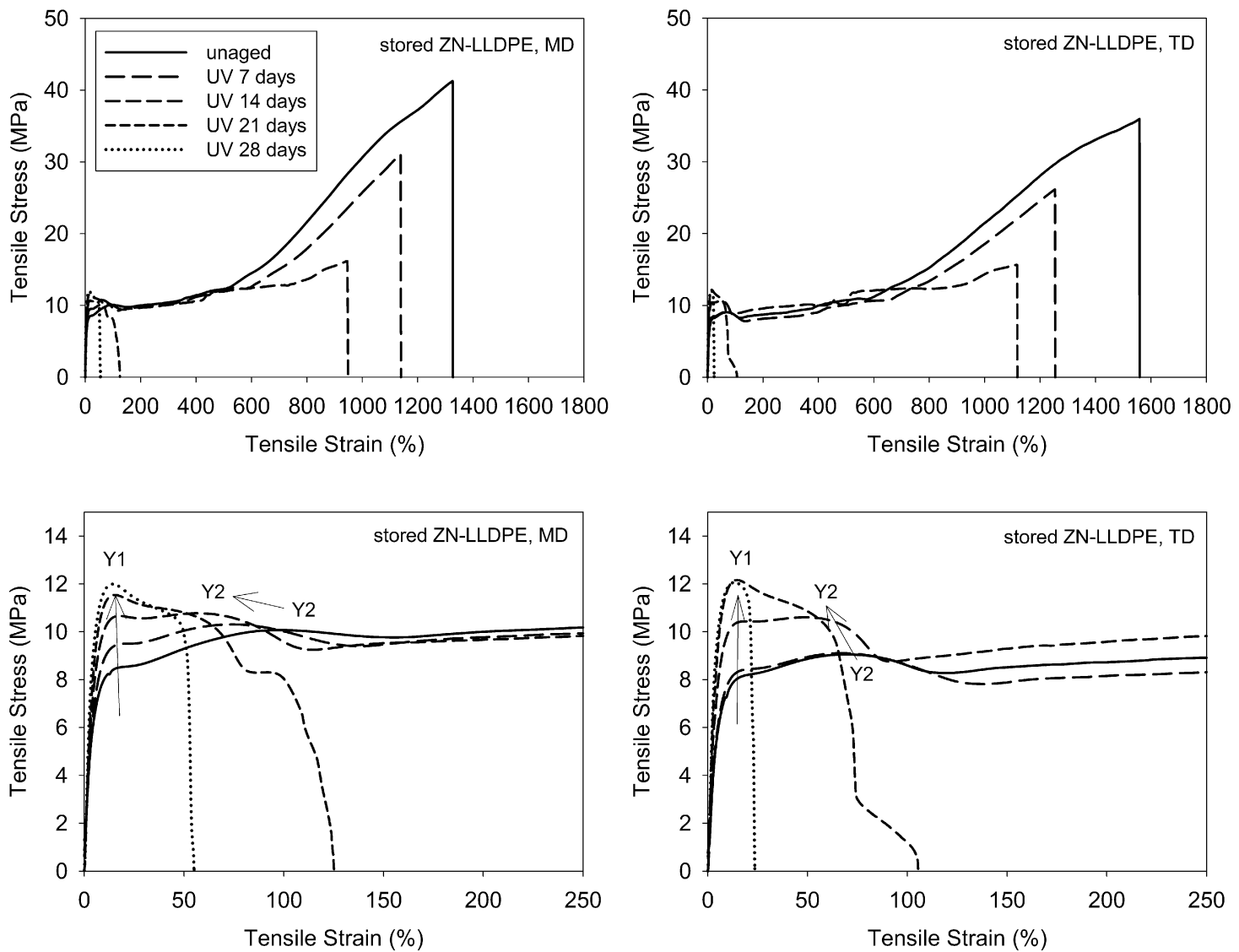

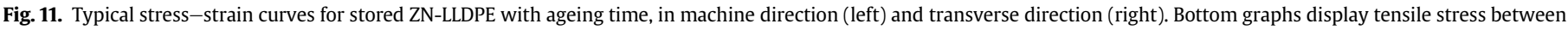
0 and $250 \%$ strain for the respective graphs in both testing direction. Y1 is the first yield point, Y2 is the second yield point, and the arrows indicate the yield point trends.

These stress-strain curves suggest that the embrittlement point was reached when the material did not pass Y2 and the ZN-LLDPE film could not sustain a stable neck. It also corresponded to when the tensile stress of Y1 was higher than Y2's.

The tensile stress of $\mathrm{Y} 1$ for ZN-LLDPE increased with ageing time, while the tensile strain of $\mathrm{Y} 1$ showed no significant change. The tensile stress of Y2 also increased but Y2's tensile strain decreased with ageing.

The first yield point in semi-crystalline polymers has been associated with the deformation of interlamellar chains, where the tensile stress acts on the tie-chain molecules and the amorphous chains surrounding lamellar crystals (Fig. 13). This effectively rotates the crystal blocks so that the chain direction is parallel to the drawing direction. When further strain was applied past $Y 1$, plastic deformation and necking begins at Y2 where the lamellar crystals start c-axis slip [13]. The stress and strain profiles for Y1 and Y2 were used as a characterisation tool to understand the changes in interlamellar and lamellar properties of photo-oxodegraded PE.

The Y1 tensile stress showed a significant increase with ageing and this increase was associated with the increase in WAXS degree of crystallinity due to recrystallisation and to a decrease in the interlamellar thickness. Both the increased crystallinity and the hindrance of more closely spaced lamellae increase the force required to rotate the lamellar crystal blocks. The tensile strain of Y1 showed no significant change, suggesting that the strain required to onset the deformation of the interlamellar phase remained the same.

The slight increase in tensile stress of Y2 with ageing was associated with an increase in crystal size (Fig. 6) (and not lamellar thickness (Fig. 9) [33]). The decrease in Y2 tensile strain with ageing suggests that the strain allowed in the interlamellar phase was restricted by the new crystals formed due to recrystallisation.

The elongation of tie molecules and deformation of the amorphous phase would occur between Y1 and Y2. The results showed that once the film had reached embrittlement, the material did not reach Y2. This suggests that taut tie molecules at the amorphous-lamellar interface were broken due to chain scission, which corresponds to the decrease in interfacial width (Fig. 10). So when these films were further drawn past Y1, c-axis slip of the lamellar crystals (associated with Y2) could not begin without the tie molecules linking the amorphous phase to the lamellar crystal (Fig. 13).

Interlamellar thinning and decrease interfacial width as a function of ageing time correlated well with the double yield behaviour and film embrittlement. It was understood that a combination of the interlamellar thickness decrease due to recrystallisation and chain scission of tie-chain molecules that eventually led to $\mathrm{ZN}$ LLDPE film transitioning from being ductile to brittle and reaching film embrittlement. Tie molecule concentration was difficult to monitor but these results demonstrated how a combination of SAXS and WAXS, and double yield point properties from the tensile stress-strain curves could give some understanding to how the interlamellar, lamellar and tie-chain molecules changed during photo-oxidative degradation.

Double yield points have been studied extensively by Brooks et al. [13], and Seguela and Rietsch [34] for (unaged) PE but they have not been studied for UV aged PE. Double yield points have shown to be a useful tool to understand the intricate crystal properties of semi-crystalline polymers. 

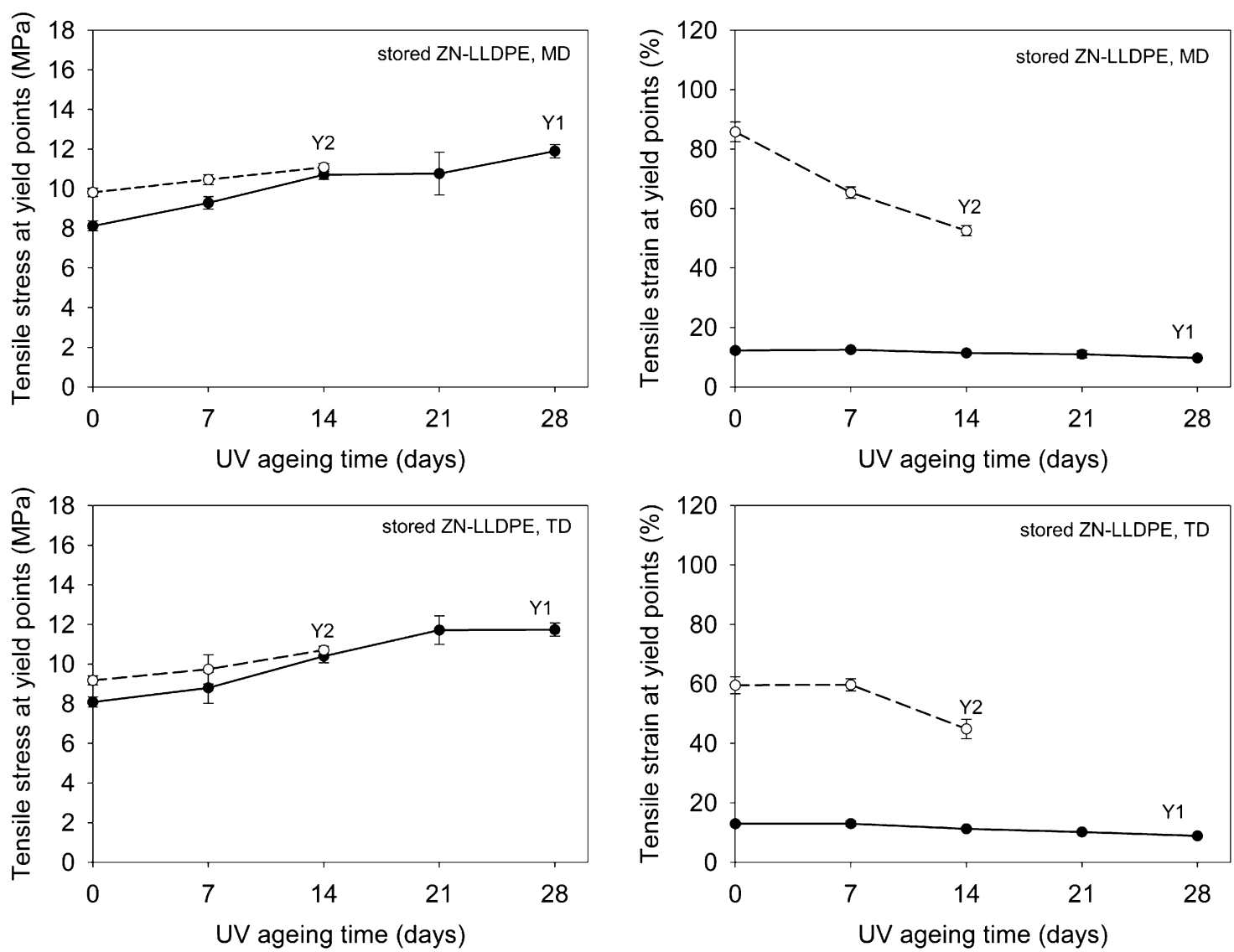

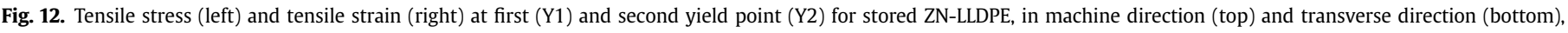
$n=8-10$, error bars $=$ standard deviation.
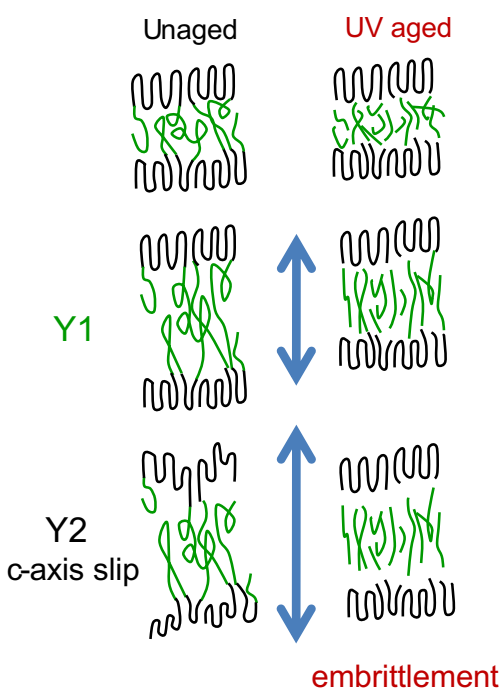

Fig. 13. Diagrams of unaged and UV aged ZN-LLDPE samples during tensile deformation up to $250 \%$ elongation - the first tensile stage depict the deformation of the interlamellar phase (onset at the first yield point (Y1)) and the c-axis slip of the lamellar crystals (onset at the second yield point (Y2)), arrows indicate the draw direction.

\section{Conclusions}

The definition of film embrittlement was not well defined in the literature. Embrittlement point was defined as the point where the elongation at break is when the film loss $95 \%$ of its initial elongation at break. This corresponded to the point where films fragmented when a small amount of force was applied. Other embrittlement criteria observed were the interlamellar thickness decreased to approximately 30-50 $\AA$ and the critical weight-average Mw at embrittlement was approximately $20,000 \mathrm{~g} / \mathrm{mol}$. These critical values were lower than Fayolle et al.'s [11] criteria (60-70 $\AA$ and 40,000-10,0000 $\mathrm{g} / \mathrm{mol}$, respectively), due to differences in embrittlement definition (50\% loss of initial elongation at break) and characterisation technique. Little correlation was seen between film embrittlement and carbonyl index, which was a result of limitations in the FTIR-ATR technique.

The mechanism in reaching film embrittlement correlated well with the mechanism proposed by Fayolle et al. [1], where chain scission reaction was the main photo-oxo-degradation mechanism observed in ZN-LLDPE films. The average molecular weight and mechanical properties decreased with UV ageing. Simultaneously, the film underwent crosslinking (shown by increased gel content), oxidation (FTIR-ATR and MAS-NMR) and recrystallisation (increased in WAXS degree of crystallinity and crystal size).

The PE chains and crystal morphology increased in heterogeneity (increased in polydispersity) with ageing time, as a result of molecular defects formed during crosslinking and the addition of bulky oxygen groups formed during oxidation.

The role of the tie molecule chains is important for the formation of a stable neck when polymer films are under tensile 
deformation. Tie-chain molecule concentration was difficult to measure directly, but the tensile results showed that as ZN-LLDPE films were exposed to UV, the film began to lose its ductile properties and it reached a point where the film could no longer strain to reach a second yield point. This signifies that the tie-chain molecules linking the lamellar crystals were scissioned and could not transmit the stress to cause c-axis slip of the lamellar crystal. This result correlated well with the decrease in interfacial width determined by SAXS data using LCF. Double yield point studies and interfacial width extracted from SAXS data, have proven to be useful methods to understand the change in tie molecule concentration and changes at the amorphous-lamellar interface as a function of UV age.

\section{Acknowledgements}

We would like to thank The University of Queensland and Cooperative Research Centre for Polymers for funding this study. Thanks to Tony McNally, Glen McClelland, Graham Garrett and John Kissick from QUB for processing these films, Samantha Passmore and Shellie Knights for their assistance on film ageing and tensile testing, Cosme Llope and Hans de Jonge from Dow for donating their time and equipment to perform the HT-GPC analysis, Ekaterina Strounina and Andrew Whittaker for performing MAS-NMR and sharing their expertise in this field. Special thanks to Graeme George for his helpful discussions. Part of this research was undertaken on the SAXS/WAXS beamline at the Australian Synchrotron, Victoria, Australia. We would like to thank Nigel Kirby, Stephen Mudie, and Adrian Hawley for their technical support at the Australian Synchrotron.

\section{References}

[1] Fayolle B, Emmanuel R, Colin X, Verdu J. J Mater Sci 2008;43:6999-7012.

[2] Jabarin SA, Lofgren EA. J Appl Polym Sci 1994;53:411-23.

[3] Roy PK, Surekha P, Rajagopal C, Chourdhary V. J Appl Polym Sci 2008;108: 2726-33.

[4] Gulmine JV, Janissek PR, Heise HM, Akcelrud L. Polym Degrad Stab 2003;79: 385-97.
[5] Guadagno L, Naddeo C, Vittoria V, Camino G, Cagnani C. Polym Degrad Stab 2001;72:175-86.

[6] Roy PK, Surekha P, Raman R, Rajagopal C. Polym Degrad Stab 2009;94: 1033-9.

[7] Ojeda T, Freitas A, Birck K, Dalmonin E, Jacques R, Bento F, et al. Polym Degrad Stab 2011;96:703-7.

[8] Kyrikou I, Briassoulis D. J Polym Environ 2007;15:125-50.

[9] Koutny M, Lemaire J, Delort AM. Chemosphere 2006;64:1243-52.

[10] Hussein IA. Polym Degrad Stab 2007;92:2026-32.

[11] Fayolle B, Colin X, Audouin L, Verdu J. Polym Degrad Stab 2007;92:231-8.

[12] Goderis B, Reynaers H, Koch MHJ, Mathot VBF. J Polym Sci B Polym Phys 1999; $1: 1715-38$.

[13] Brooks NWJ, Unwin AP, Duckett RA, Ward IM. J Macromol Sci B 1995;34: 29-54.

[14] Torikai A, Geetha R, Nagaya S, Fueki K. Polym Degrad Stab 1986;16:199-212.

[15] Miličević D, Trifunović S, Popović M, Milić TV, Suljovrujić E. Nucl Instrum Methods B 2007;260:603-12.

[16] Scott G, Gilead D. Degradable polymers. London: Chapman \& Halls; 1995.

[17] Naddeo C, Guadagno L, Luca SD, Vittoria V, Camino G. Polym Degrad Stab 2001;72:239-47.

[18] Geetha R, Torikai A, Nagaya S, Fueki K. Polym Degrad Stab 1987;19: 279-92.

[19] Liu M, Horrocks AR, Hall ME. Polym Degrad Stab 1995;49:151-61.

[20] Dow Chemical Company. Dowlex $2045 \mathrm{G}$ technical information, technical report, 2001.

[21] ASTM International. D5208, standard practice for fluorescent ultraviolet (UV) exposure of photodegradable plastics; 2007.

[22] ASTM International. D6287, standard practice for cutting film and sheeting test specimens; 2005.

[23] ASTM International. D638, standard test method for tensile properties of plastics; 2003.

[24] ASTM International. Standard test methods for determination of gel content and swell ratio of crosslinked ethylene plastics; 2006.

[25] Albertsson A, Barenstedt C, Karlsson S. Polym Degrad Stab 1992;37:163-71.

[26] Ghafor A, Halley PJ, Hill DJT, Martin DJ, Rasoul F, Whittaker AK. J Appl Polym Sci 2009;112:381-9.

[27] King S. CORFUNC overview. Oxfordshire: ISIS Facility and Diamond Light Source Ltd.. Online at: http://small-angle.ac.uk/small-angle/Software/ CORFUNC.html; 2004 (as of 25 November 2011).

[28] Peacock AJ. Handbook of polyethylene: structures, properties, and applications. New York: Marcel Dekker, Inc.; 2000.

[29] Assink RA, Celina M, Dunbar TD, Alam TM, Clough RL, Gillen KT. Macromolecules 2000;33:4023-9.

[30] Miyagawa E, Tokumitsu K, Tanaka A, Nitta K-H. Polym Degrad Stab 2007;92: 1948-56.

[31] Trojan M, Daro A, Jacobs R, David C. Polym Degrad Stab 1990;28:275-87.

[32] Munaro M, Akcelrud L. Polym Degrad Stab 2008;93:43-9.

[33] Kazmierczaka T, Galeskia A, Argon A. Polymer 2005;46:8926-36.

[34] Seguela R, Rietsch F. Polymer 1986;27:532-6. 